\title{
Rebrightening of Planetary Nebulae Through Interaction with the Interstellar Medium
}

\author{
C. J. Wareing ${ }^{\mathrm{A}}$ \\ A Department of Applied Mathematics, University of Leeds, Woodhouse Lane, Leeds, LS2 9JT, UK. \\ Email: cjw@maths.leeds.ac.uk
}

Received 2009 June 30, accepted 2009 October 9

\begin{abstract}
The interaction of planetary nebulae (PNe) with the interstellar medium (ISM) as they move through it is now acknowledged to be a major shaping effect not just for ancient and large PNe, but also for relatively young PNe with high-speed central stars. The most common effect is a rebrightening as the PN shell interacts with a pre-existing bow-shock structure formed during the previous evolutionary phase of the central star. In this review we consider this rebrightening in detail for the first time and discuss its origins, highlighting some observed examples. We go on to discuss the AGB progenitor stars, reviewing the evidence for bow-shock structures, and consider the progeny of rebrightened PNe - strongly disrupted objects which bear very little resemblance to typical PNe. Sh 2-68 is inferred to be perhaps the only documented case so far of such a PN.
\end{abstract}

Keywords: ISM: structure — planetary nebulae: general — stars: AGB and post-AGB — stars: mass-loss

\section{Introduction}

Using simple hydrogen atoms, stars forge the entire range of atomic species we observe in nature. Towards the end of their lives, stars eject these newly forged atoms into interstellar space. Out of this enriched interstellar gas, new stars, their planets and even life form. Stellar mass loss is the motor that makes stars and galaxies change over cosmic time. Yet, when and how mass is lost from stars is still an open debate. Stars more than eight times the mass of the Sun end their lives in spectacular explosions known as supernovae. Stars less than eight times the mass of the Sun lose up to $90 \%$ of their mass when they become giants, shortly after running of out hydrogen in their cores. The mass that is lost eventually forms beautiful nebulae around the mother star, objects that we call planetary nebulae; a misnomer acquired because they looked like the small circles of planets to 17 th century astronomers. Modern telescopes have revealed they exhibit complex shapes (butterfly, multiple lobes emerging from disks, jets and bullets, objects pretty enough to fill coffee table books!). These shapes are in need of an explanation: how do stars lose quite so much mass and why is this mass not residing in a more or less spherical distribution around the star?

Simple round shapes have been understood in terms of the Interacting Stellar Winds (ISW) model (Kwok 1982). In this model, planetary nebulae (PNe) are formed when stellar material surrounding the central star (CSPN), originating in a slow wind from the asymptotic giant branch (AGB) progenitor, is swept up into a nebular shell by a hot, fast, ionizing post-AGB wind from the white dwarf CSPN. The great majority of PNe are not spherical and many theories have been introduced to explain the wide variety of observed shapes. Many shapes can be reproduced in models by introducing an asymmetry into the slow wind (Kahn 1985; Balick 1987). Later theories have concentrated on the origins of the asymmetry, considering stellar rotation and/or magnetic fields (GarcíaSegura et al. 1999, 2005; Frank \& Blackman 2004). Most recently though, such effects have been shown to be difficult to maintain in single AGB stars, leaving binary central star systems as the primary progenitors of $\mathrm{PNe}$ (see, for example, De Marco 2009 for a full review and discussion of the binary progenitor hypothesis).

There are several cases though where only the outer shells show a departure from symmetry. This has been postulated to arise from an interaction with the interstellar medium (ISM). In this review, we will consider the current knowledge in this area and the previous work which has led up to the interpretation of the PN-ISM interaction as a four stage evolutionary process, including a period of 'rebrightening', typically occurring late in the lifetime of a PN. We will review this particular part of the process, including the dominating effect of the earlier AGB phase of evolution, and consider the immediate progeny of rebrightened PNe, objects which can bear little resemblance to their origins. The observational support for each stage will also be summarized here for the first time. Finally, we will discuss how rebrightening changes the lifetime estimates of typical PNe and how a sizable sample of interacting PNe may change estimates of the PN population and the understanding of ISM structure on a galactic scale. 


\section{Understanding the Interaction of PNe with the ISM}

The idea that a PN would interact with its surroundings as it moved through the ISM was first extolled by Gurzadyan (1969). Smith (1976) performed the first theoretical study in which a thin shell approximation was assumed; the 'snow-plough' momentum-conserving model of Oort (1951) was applied to shape the external shell of the PN. Isaacmann (1979) used the same approximation with higher relative velocities and ISM densities. Both concluded similarly: that a PN would fade away before any disruption of the shell by interaction with the ISM became noticeable.

The work of Borkowski, Sarazin \& Soker (1990) challenged this standpoint. They investigated CSPN with proper motions greater than $0.015 \mathrm{arcsec}_{\mathrm{yr}}^{-1}$ and found that all of the PNe showed signs of interaction with the ISM, including dipole asymmetries, distortions in the direction of the CSPN motion and displacement of the CSPN from the geometric centre of the nebula. At the same time investigating PNe with large angular extent, they found many of these also showed signs of interaction. Concluding that PN-ISM interaction must be commonplace, Borkowski, Sarazin \& Soker (1990) suggested a simple evolution whereby the PN shell is first compressed in the direction of motion and then in later stages this part of the shell is significantly decelerated with respect to the central star. They calculated that the interaction would become apparent when the densities in the expanding shell dropped below $n_{\mathrm{H}}=40 \mathrm{~cm}^{-3}$ for a PN in the Galactic Plane. As the PN fades, only the brightest regions where the shell is compressed remain detectable.

Soker et al. (1991) performed the first hydrodynamic simulations modelling the interaction, starting with the nebula shell already formed but above the upper density limit for ISM interaction to become apparent derived by Borkowski, Sarazin \& Soker (1990). They validated the thin shell approximation for calculating the displacement of CSPNs from the geometric centres of their nebulae and concluded similarly that the interaction with the ISM becomes noticeable only once the density of the shell drops below the same critical limit.

The conclusions of Borkowski, Sarazin \& Soker (1990) and Soker et al. (1991) reconciled the theory and observations, allowing for ISM interaction to become apparent once a PN had become large enough for the densities in the shell to have dropped below the critical limit, but before it faded away. At this stage though, ISM interaction was not thought to cause any increase of emission at late stages (i.e. a rebrightening), but instead to cause some areas - those interacting with the ISM — to fade more slowly than the rest of the PNe.

Soker et al. (1991) also noted that this simple picture breaks down for high velocity PNe in low density environments. Here, a Rayleigh-Taylor (RT) instability develops, leading to shell fragmentation. This fragmentation was considered in detail by Dgani \& Soker (1994) and then Dgani \& Soker (1998). In these papers, the authors performed hydrodynamic simulations of the RT instability and found that it plays an important role in the shaping of the outer regions of PN surrounding high-speed central stars. They suggested the RT instability can cause fragmentation of the nebular shell in the direction of motion. Any fragmentation caused by this instability would only be present if the CSPN relative velocity was greater than $100 \mathrm{~km} \mathrm{~s}^{-1}$. They also noted the situation could also be further complicated by the Kelvin-Helmholtz (KH) instability and magnetic fields, if inclined to the direction of motion, could break the cylindrical symmetry of the process and elongate the fragmentary structures (Dgani \& Soker 1997).

Consequently, the field reached a consensus that interaction of PNe with the ISM becomes observable only during the late stages of PN evolution and the search for evidence of the interaction became restricted to $\mathrm{PNe}$ with large angular extent (Tweedy \& Kwitter 1996; Xilouris et al. 1996; Kerber et al. 2000; Rauch et al. 2000) where it was commonly found and such PNe were classed as ancient. The definitive Atlas of Ancient PNe (Tweedy \& Kwitter 1996) found observational evidence for ISM interaction in 21 of their 27 targets.

In the first work heralding the next phase of PN-ISM modelling, Villaver, Garcia-Segura \& Manchado (2003, henceforth referred to as VGM03) pointed out that the interaction had previously been studied by considering the interaction only after the nebular shell had formed. VGM03 performed 2D hydrodynamic simulations following the preceding AGB phase of evolution, including the latest theoretical model of mass-loss variation, and found that crucially the interaction begins during that phase when the slow AGB wind is shaped by the ISM. The $\mathrm{PN}$ forms much later, initially cocooned within a bubble of undisturbed-by-ISM AGB wind material which, when the CSPN is moving through the ISM, is bounded by a bow shock formed by the AGB wind-ISM interaction and located at the point of ram pressure balance between the slow wind and the oncoming ISM. Choosing a conservative relative velocity for the CSPN, $v_{\text {ISM }}=20 \mathrm{~km} \mathrm{~s}^{-1}$ and a low density of the surrounding ISM of $n_{\mathrm{H}}=0.1 \mathrm{~cm}^{-3}$, they showed that PN-ISM interaction can become apparent at a relatively middle age with low velocities and low ISM densities. They also found the first evidence for a rebrightening, occurring when the $\mathrm{PN}$ shell has expanded far enough to interact with the AGB wind-ISM bow shock, compressing the shell in the direction of motion and increasing the density and temperature and thus the brightness. In their low-speed, low-density simulation though, this still occurred at a middle to late stage in the PN lifetime.

Showing that neither high velocities, high ISM densities, nor the presence of a magnetic field were necessary to explain the observed asymmetries in the external shells of PNe, VGM03 allowed the PN-ISM interaction to be applied to the majority of PN, not just an exceptional few. This process became important for all PNe. Their simulations also hinted that ram-pressure-stripping of mass 
downstream during the AGB and postAGB/PN phases may provide a possible solution to the problem of missing mass in PN whereby only a small fraction of the mass ejected during the AGB phase is inferred to be present during the post-AGB phase.

At the time, observational evidence for the effect of the ISM on AGB wind structures was limited. Zijlstra \& Weinberger (2002) presented evidence for a detached dust ring surrounding an AGB star, which at $4 \mathrm{pc}$ in diameter was unrivaled in size. They noted that such detached shells are normally interpreted in terms of late-AGB stage thermal pulses, however in this particular case they concluded a significant proportion of the shell may consist of sweptup ISM, with the detached appearance explained by the wind-ISM interaction.

Motivated by recent observations of the PN Sh 2-188 which revealed the true nature of the extended faint emission behind the well-known bright arc of the nebula, of which we will say more later as it is a useful example of rebrightening in PNe, Wareing, Zijlstra \& O'Brien (2007b) developed a triple-wind model in the frame of reference of the CSPN, including an initial slow AGB wind, a subsequent fast post-AGB wind, and a third continuous wind reflecting the movement through the ISM. Employing a 3D hydrodynamic scheme, they performed a range of simulations investigating the effect on PN formation of relative velocity, ISM density and CSPN mass-loss rate during the AGB and pAGB. With this extensive investigation of parameter space, they were able to generalise a four stage evolutionary classification of PN-ISM interaction, driven by the process of ram-pressure-stripping. In the next section we review the support for the idea of PN rebrightening in the context of the first three stages of this classification.

\section{Rebrightening as Part of the Four Stages of PN-ISM Interaction}

In order to demonstrate how a PN can be rebrightened, we will review the first three stages of PN-ISM interaction, according to classification of Wareing, Zijlstra \& O'Brien (2007b, henceforth referred to as WZO07). In the first stage, the PN expands inside the cocoon formed by the AGB wind-ISM bow shock formed during the preceding AGB phase, unhindered by any ISM interaction. The AGB wind-ISM interaction is radially further from the central star and it is possible to observe a faint arc of the bow shock around the PN. Such an arc is seen in several cases including the famous Dumbbell nebula, where clumps of material surrounding the bright nebula are inside what appears to be AGB wind-ISM bow shock (Meaburn et al. 2005). Early models of the interaction which did not take account of the AGB-ISM interaction could not explain the presence of this structure; an explanation required the insight of VGM03. In the case of a slow-moving star with a radially distant bow shock, WZO07 noted this stage can last for the entire PN lifetime and hence a PN-ISM interaction would never be observed. However, if the central star is moving even at average speed through the ISM (i.e. $\sim 50 \mathrm{~km} \mathrm{~s}^{-1}$,

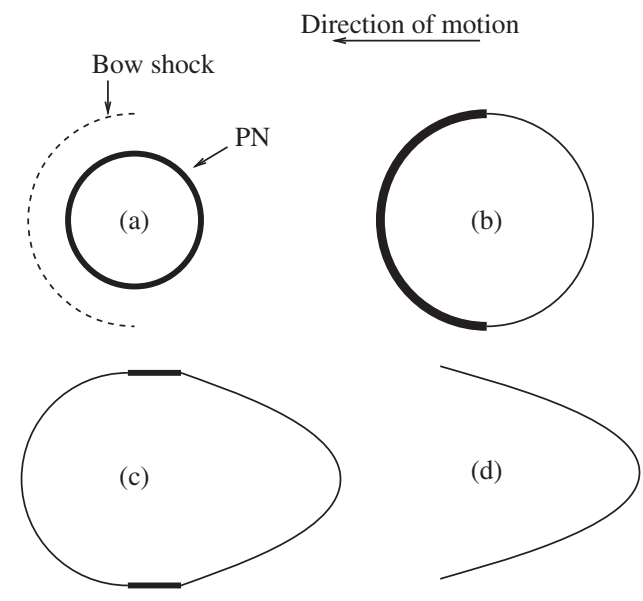

Figure 1 A reproduction of figure 7 from WZO07 showing a simple illustration of the appearance of a PN during the four stages of PN-ISM interaction. The direction of motion is to the left and thicker lines indicate the brightest regions. Panel (a) illustrates stage 1, (b) stage 2, (c) stage 3 and (d) stage 4 . The position of letters (a), (b), (c) and (d) indicates the position of the central star at each stage.

Burton 1988), then in support of VGM03, they noted PNISM interaction can rapidly occur. In some extreme cases with low CSPN mass-loss rates in high density regions, Wareing et al. noted that interaction can appear at a very young age of only 1000 years. We reproduce figure 7 from WZO07 here in Figure 1 to illustrate the primary characteristic of this stage, a swept-up ISM shell up to a few pc away.

The PN enters the second stage when it has expanded far enough to interact with the AGB wind-ISM bow shock. This is the point at which rebrightening occurs. As the expanding PN shell encounters the AGB wind bow shock in the direction of motion, it merges and drives another shock through the bow shock, increasing the density and temperature of the mixed material in that region and strengthening the emission, a rebrightening, the primary characteristic of this stage. This is illustrated in Figure $1 \mathrm{~b}$. WZO07 note that if the motion is predominantly in the plane of the sky, this part of the nebular shell may be brighter than the rest, although the PN will continue to appear circular on the sky. As the appearance deviates from circularity/symmetry and the shell is decelerated in the direction of motion by the AGB wind-ISM bow shock, rather than just interaction with the ISM as originally thought by Borkowski, Sarazin \& Soker (1990) and Soker et al. (1991), WZO07 define the PN to be entering the third stage of interaction.

This stage is characterized by the shift of geometric centre of the nebula downstream away from the CSPN as illustrated in Figure 1c. The shift, due to the deceleration of the PN shell in the direction of motion, is guaranteed to occur and provides a measurable effect of the interaction. Again, this has been noted by several authors (Borkowski, Sarazin \& Soker 1990; Soker et al. 1991; VGM03; WZO07).

The first three stages build the model which accounts for many of the characteristics of PN-ISM interaction noted by previous authors (e.g. Borkowski, Sarazin \& 
Soker 1990; Tweedy \& Kwitter 1996). In contrast to previous models though, the one-sided brightness bias observed appears to be a consequence of rebrightening, and is followed by deceleration of the shell in the direction of motion and shift of the CSPN away from the geometric centre of the nebula. We will now go on to discuss several cases of this situation, beginning with Sh 2-188 which could be considered as a template for future studies of rebrightened $\mathrm{PNe}$.

\section{Observed Cases of Rebrightened PN}

In the Atlas of Ancient PNe (Tweedy \& Kwitter 1996), Sh 2-188 was remarked upon as a bright, one-sided filamentary arc-like PN with faint, rather homogeneous material opposite the bright arc revealed by deeper $\mathrm{H} \alpha$ images. Whilst commenting on the possibility of this material being a comet-like tail, the authors opted for the simpler explanation that Sh 2-188 resides in a highly inhomogeneous ISM. 2003 observations, taken as part of the Isaac Newton Group Photometric $\mathrm{H} \alpha$ Survey of the Northern Galactic Plane (IPHAS) (Drew et al. 2005) revealed the faint material to be a ring-like completion of the arc and a tail stretching away in opposition to the bright arc. Wareing et al. (2006a) modelled the nebula as a strong PN-ISM interaction where the CSPN is moving at $125 \mathrm{~km} \mathrm{~s}^{-1}$ in the direction of the bright arc relative to the ISM. In Figure 2, we reproduce their figure 3 combining the IPHAS observations of the nebula. The nebular shell is interacting with the AGB wind-ISM bow shock and undergoing the exact rebrightening discussed in the previous section. Sh 2-188 is a prime example then of the effect under discussion in this review. The faint completion of the arc is the original nebular shell continuing to expand into AGB wind material cocooned by the bow shock and tail. The tail consists of far-older ram-pressure-stripped material from the head of the bow shock stretching away to the North-West.

Wareing et al. (2006a) note that if the central star had not been traveling through the local ISM, Sh 2-188 would most likely have a ring-like appearance on the sky with a radius similar to the distance between the faint completion of the arc and the CSPN - approaching twice the actual radius. The surface brightness of the whole object would be comparable to the faint completion of the ring. Clearly, ISM interaction has strongly affected the late evolution of this particular nebula, limiting its expansion and rebrightening the nebula on the upstream side of the shell. Finally, Wareing et al. (2006a) performed a proper motion analysis and found the CSPN to have a considerable motion of $0.03 \pm 0.01 \operatorname{arcsec}_{\mathrm{yr}}-1$ in the direction of the bright arc, confirming the PN-ISM interaction hypothesis. The combination of the proper motion and the high velocity required to model the appearance of the nebula, placed it at distance of $850 \mathrm{pc}$, consistent with other estimates of the distance to the star.

The 21 candidate PN-ISM interactions in the Atlas of Interacting PNe appear to show similar rebrightening characteristics. Of those, Sh 2-216 shows filamentary evidence of a particularly complex magnetized ISM in the

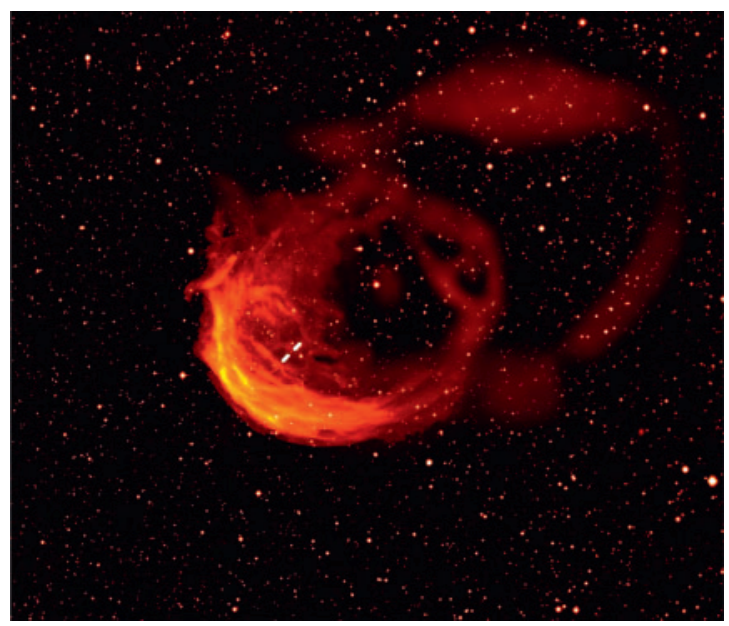

Figure 2 A reproduction of figure 3 from Wareing et al. (2006a) showing a combination of observations of the PN Sh 2-188 taken as part of IPHAS. The faint central star is indicated on the image between the markers. In the image, North is up and East to the left.

vicinity of the PN (Ransom et al. 2008). Several objects in the search for extensive halos around PNe (Hao Hsia, Zeng Li \& Ip 2007) also show evidence for one-sided rebrightening, suggesting the interaction is commonplace. There is also the complex case of the bipolar nebulosity surrounding the nova GK Per: thought to be an ancient PN ejected by a member of the binary system, the nebula has a swept back appearance, brightened on the upstream side, in alignment with the proper motion of the system (Bode, O'Brien \& Simpson 2004). The MASH (Parker et al. 2006) and MASH-II (Miszalski et al. 2008) catalogues are now beginning to reveal many more cases of interacting PNe e.g. PFP1 (Pierce et al. 2004).

Evidence for other PNe with tails is considerably rarer. Deep $\mathrm{H} \alpha+\mathrm{N}$ II observations of HFG1 (Xilouris et al. 1996; Boumis et al. 2009) reveal a strong candidate tail. Szentgyorgyi et al. (2003) find the emission of [SII] in the PN NGC 246 probably traces the interaction of the PN with the ISM. Meaburn et al. (2000) find mid-IR emission apparently tracing a tail behind NGC 3242, supported by Ramos-Larios \& Phillips (2009) who find evidence for ram-pressure stripped material in two other PNe NGC 2440 and NGC 6629. Finally, the complex and asymmetric ancient PN Sh 2-68 appears to have evidence for a long tail, stretching away from the location of the CSPN in opposition to the direction of proper motion (Xilouris et al. 1996). This object may be the first documented case of the progeny of a rebrightened PN, but first we will discuss the characteristics of the AGB progenitors, and the evidence in the literature for such objects.

\section{The Progenitors of Rebrightened PNe}

The AGB progenitors of ISM-interaction-rebrightened PNe must be moving through the ISM at considerable velocities. Wareing et al. (2006a) hypothesized that bow shock-like structures must exist around such progenitor stars. This prediction was rapidly borne out in observations of the AGB star R Hya. Recent IR observations of 
the star, taken as part of the MIRIAD programme, revealed an arc-like structure to the North-West of the star (Ueta et al. 2006), aligned in the direction of the star's proper motion. Wareing et al. (2006b) modelled this structure as an AGB wind-ISM bow shock ahead of the star, confirming the existence of such structures and showing that the dominant shaping factor for PN-ISM interaction and the origin of PNe rebrightening is formed during the preceding AGB phase of evolution.

Evidence for similar bow shocks has now been found around several other giant and AGB stars, e.g. RX Lep (Libert et al. 2008) and R Cas (Ueta et al. 2009). In the case of the famous red giant $\alpha$ Orionis, observations presented by Noriega-Crespo et al. (1997) revealed an arc-like structure through a novel data-reduction technique. The observations constitute what is probably the earliest evidence for a late-evolution stellar wind-ISM bow shock, recently confirmed and analytically modelled by Ueta et al. (2008). None of these cases, including that of R Hya, found any clear evidence for a tail of ram-pressure-stripped material behind the bow shock.

Definitive evidence of such a tail was fortuitously discovered behind the famous Mira binary system as part of routine GALEX ultraviolet observations, originating from the AGB star Mira A (Martin et al. 2007). The narrow collimated tail stretches 2 degrees on the sky, equivalent to $4 \mathrm{pc}$ at the distance of the binary system, behind an arc-like structure ahead of the system. We reproduce an image of the structure in Figure 3. Wareing et al. (2007a) modelled the structure as a bow shock and ram-pressure-stripped tail stretching away from the location of the binary system. For a full discussion of Mira's tail and its characteristics, we review the reader to the recent review of Wareing (2008). Close to the binary system, there is evidence for asymmetric outflow (Meaburn et al. 2009). This recent outflow ( $\sim 1000$ years old), whilst not aligned with the tail, will strongly affect the PN that will eventually form around Mira A. The most-likely bipolar PN will then later experience a complex rebrightening as it interacts with the bow shock. Given the relatively high speed and close proximity of the bow shock to the binary system, the simulations of WZO07 suggest Mira's PN will relatively rapidly interact with the ISM. Mira and its tail are a template progenitor of PN-ISM interaction.

\section{The Progeny of Rebrightened PNe: Implications for Future Observational Work}

The four stage classification of WZO07 predicts the future of rebrightened $\mathrm{PNe}$ in the final stage. At this time, their model predicts a deceleration of the shell in the direction of motion combined with ram-pressure-stripping of material, driving the majority of nebular material into the tail. The fast wind from the CSPN has formed another bow shock, much closer to the CSPN. The remaining AGB wind material and the remnants of the $\mathrm{PN}$ shell are swept downstream in turbulent regions of high density and temperature as illustrated in Figure 1d. Any observable structure would be difficult to identify as a PN.

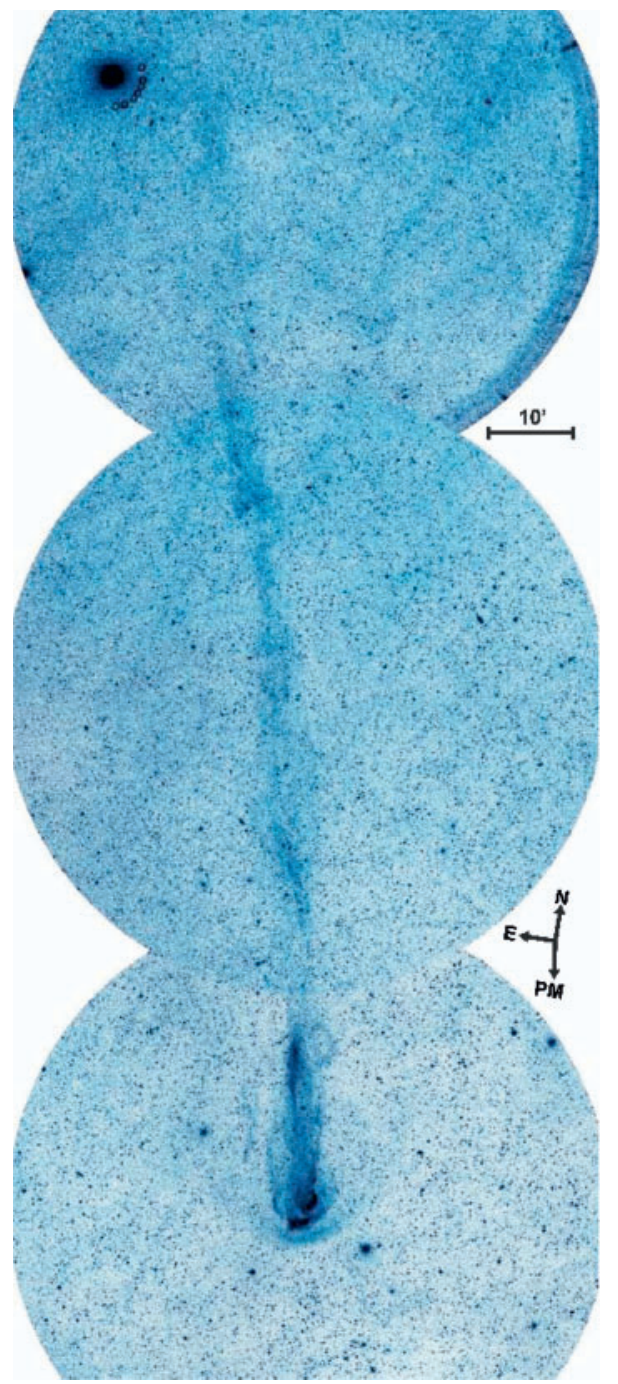

Figure 3 A reproduction of figure $1 \mathrm{~b}$ from Wareing et al. (2007a) showing the 2-degree structure stretching behind the location of the Mira binary system.
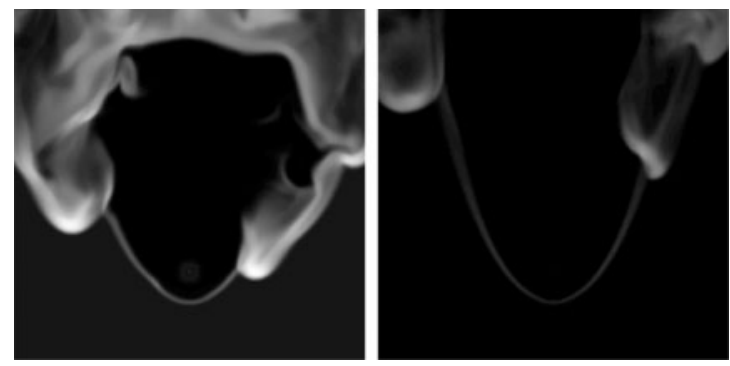

Figure 4 Panels showing gas density during the PN phase for a PN moving at a relative speed of $125 \mathrm{~km} \mathrm{~s}^{-1}$. On the left we show a slice through the gas density datacube at the position of the central star, perpendicular to the direction of motion, at 15000 years into the PN phase, on the right at 20000 years into the PN phase. The slices are $1 \mathrm{pc}$ on a side.

We are able to show two snapshots of this evolution in Figure 4. The snapshots are taken from the Case E simulation presented in WZO07. They are at later times, 15000 and 20000 years into the PN phase, than the snapshots presented in that work. Moving at $125 \mathrm{~km} \mathrm{~s}^{-1}$ through an 
average galactic plane ISM density of $n_{\mathrm{H}}=2 \mathrm{~cm}^{-3}$, the PN has long since entered the fourth stage of interaction. The fast wind bow shock has formed against the oncoming ISM and the remains of the nebular shell and AGB wind bow shock are being swept downstream in turbulent regions of high density and temperature.

There are very few possible detections of such objects in the literature and given their likely advanced age and high speed central stars, this comes as no surprise. The primary example is Sh 2-68, a nebula that shows all the signs of being swept downstream behind the central star. The CSPN has one of the largest measured proper motions:

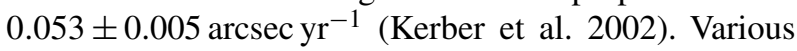
distance estimates exist to the nebula, but range over 500 $1000 \mathrm{pc}$ (Napiwotzki \& Schoenberner 1991, 1995). At this distance, the CSPN is likely at the very high speed end of the CSPN velocity range (Burton 1988), around $150 \mathrm{~km} \mathrm{~s}^{-1}$. The filamentary structure of the nebula is attributed by Kerber et al. to a Rayleigh-Taylor instability, which fits well with previous theoretical studies (Dgani \& Soker 1994, 1998). However, the filaments are all aligned with the direction of motion and they could simply be the rebrightened regions of high density and temperature predicted by the four-stage model being ram-pressurestripped into the tail. In particular, the strongest emission from Sh 2-68 comes from a long arc, at the edge of the emission and 'pointing' in the direction of the CSPN. We show an image of the nebula in Figure 5. This fits well with the structures shown in Figure 4. Note also that faint material ahead of the star appears to be confined by the inferred position of the ancient bow shock. Interestingly, Xilouris et al. (1996) found a extended halo around Sh 2-68 in the form of a cometary tail in opposition to the direction of proper motion. If this material is connected to the nebula, and the alignment with the direction of proper motion strongly supports this, then Sh 2-68 is the key example of the fate of rebrightened PN and deserving of future investigation.

Large, faint objects such as Sh 2-68 and Sh 2-188 are now regularly being observed as part of sky surveys, e.g. IPHAS, VPHAS, MASH-II, and new astronomical tools are able to take deeper observations, e.g. the Herschel Space Telescope will soon begin its survey of cold dust around evolved stars and is likely to reveal many more tails such as the one behind Mira. These surveys and instruments are likely to reveal the ancient, faint, extended progenies of rebrightened $\mathrm{PNe}$, such as Sh 2-68. The structures will have little resemblance to young PN, their central stars may be far from the strongest emission and any remnants of nebular shells are likely to have been long since swept into turbulent regions in the tail. Identification of candidate CSPN will be possible by proper motion studies - elements of the structure are highly likely to be a aligned with the direction of motion. These objects will have to be carefully filtered and confirmations of a candidate stage WZO 4 PN revealed by IPHAS are already proving difficult (L. Sabin, private communication; cf. Sabin's paper elsewhere in this issue).

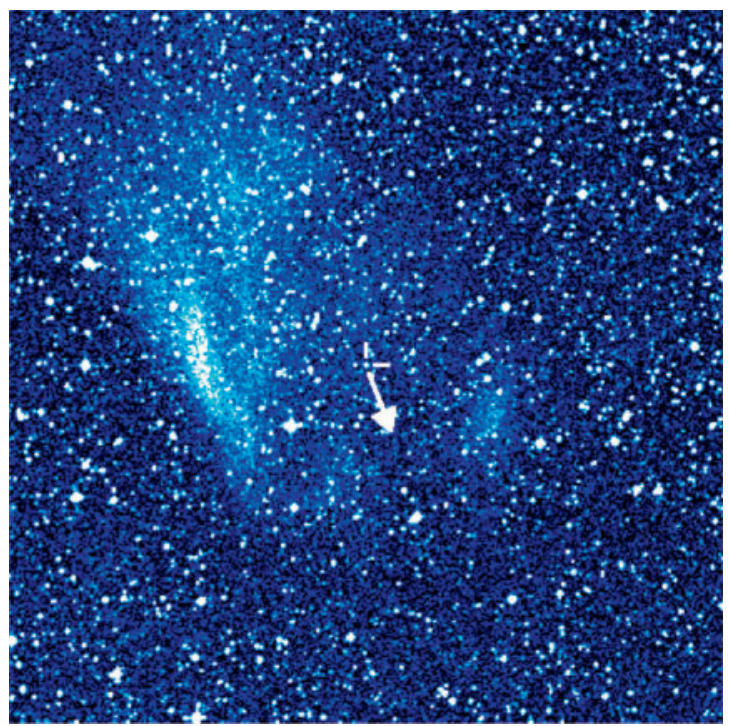

Figure 5 A $15^{\prime} \times 15^{\prime}$ image of the PN Sh 2-68. The CSPN is indicated between the markers and the arrow indicates the direction of the proper motion; $202 \mathrm{deg} \pm 6 \mathrm{deg}$ East of North Kerber (2002). In the image, North is up and East to the left. The image has been retrieved from the digitised SuperCOSMOS H $\alpha$ survey of the Southern Galactic Plane (Parker et al. 2005).

\section{Concluding Remarks}

Simulations have shown that interaction with the ISM strongly affects the death of a PN, even one with an average velocity central star. The structure of the AGB wind around the CSPN is changed from a wall into a bow shock during the preceding phase of evolution. This bow shock and the ram pressure stripping of material into the accompanying tail then dominates the evolution of the PN. Rebrightening and rejuvenation occur, on a short timescale for high speed CSPN, as the PN shell interacts with the bow shock. Following this, the $\mathrm{PN}$ is stripped downstream in turbulent regions moving down the tail, resulting in the central star leaving its PN.

Further study of rebrightened PN and their surroundings can provide considerable information regarding several other research avenues. As highlighted in the opening paragraph, tracing the ejected mass in circumstellar structures such as bow shocks, tails and late-evolution turbulent regions swept down the tail can reveal much about the way AGB and post-AGB stars lose mass. Such information as the position of the bow shock and the parameters of the tail can also reveal, taking into account the method of formation, not only historical stellar mass-loss rates and local ISM conditions, but also details of galactic orbits for the first time. The few objects that have observed lengthy tails, including Mira, HFG1 and Sh 2-68, are currently uniquely placed to derive such orbits. Future surveys of cool dust around AGB stars should reveal many more tails allowing a deeper investigation of a meaningful sample.

Once we have a meaningful sample of rebrightened $\mathrm{PNe}$, we should also be able to see how the observable lifetimes of PNe are extended. Current statistical population estimates, which depend on estimated lifetimes, indicate a 
larger population than we have currently detected, but by extending that lifetime, population estimates accordingly reduce and we are likely to see much more of an agreement between the numbers we actually observe, which is believed to be fairly complete within the Milky Way, and the predicted population.

The model of PN-ISM interaction can account for many characteristics of PNe and explain highly complex, disrupted objects such as Sh 2-68, but it does not reproduce several observed details. In particular, the fragmentation of the nebular rim is not reproduced by the simulations. More advanced models must be able to reproduce this, at least in the cases of high speed CSPN as it seems common at this end of the velocity distribution, e.g. Sh 2-188. No theoretical investigation has yet been performed employing 3D MHD simulations which include the galactic magnetic field. This would seem a natural next step and as Dgani and Soker point out, may rapidly provide an explanation of particularly fragmentary shells. In a greater challenge to the model, the existence of ancient, effectively spherical PNe with very few signs of ISM interaction are difficult to explain within the model context, especially if they have displaced central stars which rules out an alignment between viewing angle and direction of motion. The model of PN-ISM interaction has come along way and revealed the phenomenon of rebrightened PNe, but there are a still a number of open questions to be solved and no doubt future observations will reveal many more.

\section{Acknowledgments}

The author acknowledges and thanks several researchers who's input has greatly helped in this project and the creation of this review paper including Prof. A. A. Zijlstra, Assoc. Prof. O. De Marco, Dr T. J. O'Brien, Dr M. Lloyd and Dr L. Sabin. C.J.W. is funded through a post-doctoral Research Fellowship supported by the Science \& Technology Facilities Council (grant number PP/ E001092/1).

\section{References}

Balick, B., 1987, AJ, 94, 671

Bode, M. F., O’Brien, T. J. \& Simpson, M., 2004, ApJ, 600, L63 Borkowski, K. J., Sarazin, C. L. \& Soker, N., 1990, ApJ, 360, 173

Boumis, P., Meaburn, J., Lloyd, M. \& Akras, S., 2009, MNRAS, 396, 1186

Burton, W. B., 1988, in Galactic and Extragalactic Radio Astronomy, Eds. Kellermann, K. \& Verschuur, G. L. (New York: Springer), 295

De Marco, O., 2009, PASP, 121, 316

Dgani, R. \& Soker, N., 1994, ApJ, 434, 262

Dgani, R. \& Soker, N., 1997, ApJ, 484, 277

Dgani, R. \& Soker, N., 1998, ApJ, 495, 337

Drew, J. E. et al., 2005, MNRAS, 362, 753

Frank, A. \& Blackman, E. G., 2004, ApJ, 614, 737
García-Segura, G., Langer, N., Różyczka, M. \& Franco, J., 1999, ApJ, 517, 767

García-Segura, G., López, J. A. \& Franco, J., 2005, ApJ, 618, 919

Gurzadyan, G. A., 1969, Planetary Nebula (New York: Gordon \& Breach), 235

Hao Hsia, C., Zeng Li, J. \& Ip, W.-H., 2007, eprint (astro-ph/ 0712.2639)

Isaacmann, R., 1979, A\&A, 77, 327

Kahn, F. D. \& West, K. A., 1984, MNRAS, 212, 837

Kerber, F., Furlan, E., Rauch, T. \& Roth, M., 2000, ASPC, 199, 313

Kerber, F., Guglielmetti, F., Mignani, R. \& Roth, M., 2002, A\&A, 381, L9

Kwok, S., 1982, AJ, 258, 280

Libert, Y., Le Bertre, T., Gérard, E. \& Winters, J. M., 2008, A\&A, 491, 789

Martin, D. C. et al., 2007, Nature, 448, 780

Matthews, L. D., Libert, Y., Gérard, E., Le Bertre, T. \& Reid, M. J., 2008, ApJ, 684, 603

Miszalski, B., Parker, Q. A., Acker, A., Birkby, J. L., Frew, D. J. \& Kovacevic, A., 2008, MNRAS, 384, 525

Meaburn, J., López, J. A. \& Noriega-Crespo, A., 2000, ApJS, 128,321

Meaburn, J., Boumis, P., Christopoulou, P. E., Goudis, C. D., Bryce, M. \& López, J. A., 2005, RMxAA, 41, 109

Meaburn, J., López, J. A., Boumis, P., Lloyd, M. \& Redman, M. P., 2009, A\&A, 500, 827

Napiwotzki, R. \& Schoenberner, D., 1991, A\&A, 249, L16

Napiwotzki, R. \& Schoenberner, D., 1995, A\&A, 301, 545

Noriega-Crespo, A., van Buren, D., Cao, Y. \& Dgani, R., 1997, AJ, 114,837

Oort, J. H., 1951, Problems of Cosmical Aerodynamics (Dayton: Central Air Document Office)

Parker, Q. A. et al., 2005, MNRAS, 362, 689

Parker, Q. A. et al., 2006, MNRAS, 373, 79

Pierce, M. J., Frew, D. J., Parker, Q. A. \& Köppen, J., 2004, PASA, 21,334

Ramos-Larios, G. \& Phillips, J. P., 2009, MNRAS, 400, 575

Ransom, R. R., Uyaniker, B., Kothes, R. \& Landecker, T. L., 2008, ApJ, 684, 1009

Rauch, T., Furlan, E., Kerber, F. \& Roth, M., 2000, ASPC, 199, 341

Smith, H., 1976, MNRAS, 175, 419

Soker, N., Borkowski, K. J. \& Sarazin, C. L., 1991, AJ, 102, 1381

Soker, N., 1996, ApJ, 469, 734

Szentgyorgyi, A., Raymond, J., Franco, J., Villaver, E. \& LópezMartin, L., 2003, 594, 874

Tweedy, R. W. \& Napiwotzki, R., 1994, AJ, 108, 978

Tweedy, R. W. \& Kwitter, K. B., 1996, ApJS, 107, 255

Ueta, T. et al., 2006, ApJ, 648, L39

Ueta, T., 2008, ApJ, 687, L33

Ueta, T. et al., 2008, PASJ, 60, S407

Ueta, T. et al., 2009, eprint (astro-ph/0905.0750)

Villaver, E., Garcia-Segura, G. \& Manchado, A., 2003, ApJ, 585, L53 [VGM03]

Wareing, C. J. et al., 2006a, MNRAS, 366, 387

Wareing, C. J. et al., 2006b, MNRAS, 372, L63

Wareing, C. J., Zijlstra, A. A., O’Brien, T. J. \& Seibert, M., 2007a, ApJ, 670, L125

Wareing, C. J., Zijlstra, A. A. \& O’Brien, T. J., 2007b, MNRAS, 382, 1233 [WZO07]

Wareing, C. J., 2008, RSPTA, 366, 4429

Xilouris, K. M., Papamastorakis, J., Paleologou, E. \& Terzian, Y., 1996, A\&A, 310, 603

Zijlstra, A. A. \& Weinberger, R., 2002, ApJ, 572, 1006 